This item was submitted to Loughborough's Research Repository by the author.

Items in Figshare are protected by copyright, with all rights reserved, unless otherwise indicated.

\title{
Asymptotics of scaling parameters for period-doubling in unimodal maps with asymmetric critical points
}

PLEASE CITE THE PUBLISHED VERSION

\section{LICENCE}

CC BY-NC-ND 4.0

\section{REPOSITORY RECORD}

Mestel, B.D., and Andrew H. Osbaldestin. 2019. "Asymptotics of Scaling Parameters for Period-doubling in Unimodal Maps with Asymmetric Critical Points”. figshare. https://hdl.handle.net/2134/811. 


\title{
Asymptotics of scaling parameters for period-doubling in unimodal maps with asymmetric critical points
}

\author{
B D Mestel \\ School of Mathematical Sciences \\ University of Exeter \\ Exeter \\ EX4 4QE, UK \\ A H Osbaldestin \\ Department of Mathematical Sciences \\ Loughborough University \\ Loughborough \\ LE11 3TU, UK
}

November 19, 1998

\begin{abstract}
The universal period-doubling scaling of a unimodal map with an asymmetric critical point is governed by a period-2 point of a renormalisation operator. The period-2 point is parametrised by the degree of the critical point and the asymmetry modulus. In this paper we study the asymptotics of period- 2 points and their associated scaling parameters in the singular limit of degree tending to 1.
\end{abstract}

\section{Introduction}

In this paper we study the asymptotics of the solutions of the following functional equations:

$$
\begin{aligned}
& \tilde{f}_{L}(x)=-\lambda^{-1} f_{R} f_{R}(-\lambda x), \\
& \tilde{f}_{R}(x)=-\lambda^{-1} f_{R} f_{L}(-\lambda x), \\
& f_{L}(x)=-\tilde{\lambda}^{-1} \tilde{f}_{R} \tilde{f}_{R}(-\tilde{\lambda} x), \\
& f_{R}(x)=-\tilde{\lambda}^{-1} \tilde{f}_{R} \tilde{f}_{L}(-\tilde{\lambda} x),
\end{aligned}
$$

with the normalisations $f_{L}(0)=f_{R}(0)=\tilde{f}_{L}(0)=\tilde{f}_{R}(0)=1$ so that $\lambda=-f_{R}(1)>0$ and $\tilde{\lambda}=-\tilde{f}_{R}(1)>0$.

These equations arise in the theory of period-doubling cascades for families of unimodal maps $f$ of an interval with a single critical point of degree $d$ and at which the left and right $d$ th derivative at 0 differ.

In [19] it was shown that the critical behaviour of such families of maps was governed by a period-two point of a generalised Feigenbaum renormalisation operator. The period-two 
point is given by the solution of the functional equations (1.1). (Note that these equations were written with $\alpha=-\lambda$ and $\tilde{\alpha}=-\tilde{\lambda}$ in [19].)

Here $f_{L}$ and $f_{R}$ correspond to the left and the right parts of the unimodal map $f$, i.e.,

$$
f(x)= \begin{cases}f_{L}(x) & \text { if } x \leq 0 \\ f_{R}(x) & \text { if } x \geq 0\end{cases}
$$

The solutions of (1.1) depend on two parameters, viz., the degree $d$ of the critical point and the asymmetry 'modulus' $\mu$, which (for the case when $d$ is an even integer) is the ratio

$$
\mu=\frac{f_{L}^{(d)}(0-)}{f_{R}^{(d)}(0+)} .
$$

When $\mu=1$, the equations (1.1) correspond to the standard Feigenbaum equation

$$
f(x)=-\lambda^{-1} f f(-\lambda x), \quad f(0)=1 .
$$

In [20] the Herglotz function techniques of $\mathrm{H}$ Epstein $[9,10,11]$ were adapted to prove the existence of a solution of the equations (1.1) for all real $\mu>0$ and $d>1$.

It should be noted that the fixed-point theorem used in [20] to prove existence of a solution of (1.1) does not guarantee uniqueness. It is however believed that analytic solutions of (1.1) are unique up to normalisation.

In this paper we study the asymptotics of the solutions of the equations (1.1) in the limit $d \rightarrow 1+$. We are particularly interested in the scaling parameters $\lambda, \tilde{\lambda}$. This work follows on from previous studies of the asymptotics of the solutions of the Feigenbaum equation (1.4) in the limit $d \rightarrow 1+[2]$.

Our results may be summarised as follows:

Theorem. For fixed $\mu>1$, there exists a family of solutions to (1.1) parametrised by $d>1$ satisfying in the limit $d \rightarrow 1+$

$$
\begin{aligned}
& \lambda \sim C \mu^{-1 /(2(d-1))} \\
& \tilde{\lambda} \sim \sqrt{\mu}-1,
\end{aligned}
$$

where

$$
C=\frac{\mu^{(1+2 \sqrt{\mu}) /(4(\sqrt{\mu}-1))}}{e^{3}(\sqrt{\mu}-1)^{1 / 2}} .
$$

The theorem will be proved in Sections 3 and 4. A corresponding result for $\mu<1$ may be obtained by switching $\lambda$ and $\tilde{\lambda}$ in the above theorem.

This result differs substantially from the symmetric case $\mu=1$. In [2] it is shown that for $\mu=1$ there exist solutions with

$$
\lambda=\tilde{\lambda} \sim-(d-1) \log (d-1),
$$

as $d \rightarrow 1+$.

\section{The Herglotz function approach}

The Herglotz function approach as pioneered by Epstein [9] has been an extremely fruitful technique in the analysis of the accumulation of period-doubling. For the problem in hand, 
it was used in [20] to prove the existence of a solution of the equations (1.1) for all real $\mu>0$ and $d>1$. We recall here how equations (1.1) may be recast as an anti-Herglotz function problem.

Firstly we build the singularity into our functions by defining

$$
f_{R}(x)=F_{R}\left(|x|^{d}\right), \quad \tilde{f}_{R}(x)=\tilde{F}_{R}\left(|x|^{d}\right) .
$$

The left-hand functions are given in terms of the right-hand ones by

$$
f_{L}(x)=F_{L}\left(|x|^{d}\right)=F_{R}\left(\mu|x|^{d}\right), \quad \tilde{f}_{L}(x)=\tilde{F}_{L}\left(|x|^{d}\right)=\tilde{F}_{R}\left(\mu^{-1}|x|^{d}\right) .
$$

We then we consider the inverses of these functions by defining

$$
F_{R}(x)=U^{-1}(x), \quad \tilde{F}_{R}(x)=\tilde{U}^{-1}(x) .
$$

The functions $U$ and $\tilde{U}$ satisfy

$$
\begin{array}{ll}
U(1)=0, & U(-\lambda)=1, \\
\tilde{U}(1)=0, & \tilde{U}(-\tilde{\lambda})=1 .
\end{array}
$$

To finish the transformation we normalise by setting

$$
U(x)=k \psi(x), \quad \tilde{U}(x)=\tilde{k} \tilde{\psi}(x),
$$

where

$$
k=U(0), \quad \tilde{k}=\tilde{U}(0),
$$

so that the functions $\psi$ and $\tilde{\psi}$ satisfy

$$
\begin{array}{ll}
\psi(1)=0, & \psi(0)=1, \\
\tilde{\psi}(1)=0, & \tilde{\psi}(0)=1 .
\end{array}
$$

These normalisation constants will be of great use to us in what follows, and we may write this last step as

$$
U(x)=z_{1}^{d} \psi(x), \quad \tilde{U}(x)=\tilde{z}_{1}^{d} \tilde{\psi}(x)
$$

where

$$
z_{1}=\psi(-\lambda)^{-1 / d}, \quad \tilde{z}_{1}=\tilde{\psi}(-\tilde{\lambda})^{-1 / d}
$$

In this new setting our equations are

$$
\psi(x)=\frac{\mu \tilde{z}_{1}^{d}}{\tilde{\lambda}^{d} z_{1}^{d}} \tilde{\psi}\left(\tilde{z}_{1} \tilde{\psi}(-\tilde{\lambda} x)^{1 / d}\right), \quad \tilde{\psi}(x)=\frac{z_{1}^{d}}{\mu \lambda^{d} \tilde{z}_{1}^{d}} \psi\left(z_{1} \psi(-\lambda x)^{1 / d}\right) .
$$

These may be written in the form

$$
\psi(x)=\tilde{\tau}^{-1} \tilde{\psi}(\tilde{\phi}(x)), \quad \tilde{\psi}(x)=\tau^{-1} \psi(\phi(x)),
$$

where

$$
\phi(x)=z_{1} \psi(-\lambda x)^{1 / d}=\frac{\psi(-\lambda x)^{1 / d}}{\psi(-\lambda)^{1 / d}}, \quad \tilde{\phi}(x)=\tilde{z}_{1} \tilde{\psi}(-\tilde{\lambda} x)^{1 / d}=\frac{\tilde{\psi}(-\tilde{\lambda} x)^{1 / d}}{\tilde{\psi}(-\tilde{\lambda})^{1 / d}},
$$


and

$$
\tau=\psi\left(z_{1}\right)=\psi\left(\psi(-\lambda)^{-1 / d}\right), \quad \tilde{\tau}=\tilde{\psi}\left(\tilde{z}_{1}\right)=\tilde{\psi}\left(\tilde{\psi}(-\tilde{\lambda})^{-1 / d}\right)
$$

satisfy

$$
\lambda^{d}=\frac{\tau z_{1}^{d}}{\mu \tilde{z}_{1}^{d}}, \quad \tilde{\lambda}^{d}=\frac{\mu \tilde{\tau} \tilde{z}_{1}^{d}}{z_{1}^{d}} .
$$

The method of the existence proof is now to show that (2.10) has a solution in a suitably chosen space of pairs of functions.

Definition. Let $\mathbb{C}_{+}, \mathbb{C}_{-}$denote the upper and lower half planes in $\mathbb{C}$. A complex analytic function on $\mathbb{C}_{+} \cup \mathbb{C}_{-}$is said to be Herglotz (resp. anti-Herglotz) if $f\left(\mathbb{C}_{+}\right) \subseteq \overline{\mathbb{C}}_{+}$and $f\left(\mathbb{C}_{-}\right) \subseteq$ $\overline{\mathbb{C}}_{-}\left(\right.$resp. $f\left(\mathbb{C}_{+}\right) \subseteq \overline{\mathbb{C}}_{-}$and $\left.f\left(\mathbb{C}_{-}\right) \subseteq \overline{\mathbb{C}}_{+}\right)$.

Let $A, B \in \mathbb{R}$ satisfy $A<0<1<B$ and let $\Omega(A, B)$ denote $\mathbb{C}_{+} \cup \mathbb{C}_{-} \cup(A, B)$. We denote by $\mathrm{H}(A, B)$ and $\mathrm{AH}(A, B)$ (respectively) the space of Herglotz and anti-Herglotz functions (respectively) analytic on the interval $(A, B)$. Furthermore, let $\mathrm{E}(A, B)$ denote the space of anti-Herglotz functions $\psi \in \mathrm{AH}(A, B)$ which satisfy the normalisations

$$
\psi(0)=1, \quad \psi(1)=0 .
$$

As is normal, we equip $\mathrm{H}(A, B), \mathrm{AH}(A, B)$ and $\mathrm{E}(A, B)$ with the topology of uniform convergence on compact subsets of $\Omega(A, B)$.

Herglotz and anti-Herglotz functions have a number of important properties which we list here (see [9] and references therein):

1. $\mathrm{E}(A, B)$ is compact;

2. Any non-constant function $f \in \mathrm{H}(A, B)$ (resp. $\mathrm{AH}(A, B)$ ) is strictly increasing (resp. decreasing) on any open interval of $\mathbb{R}$ on which it is analytic; moreover, on such an interval, the Schwarzian derivative $S(f)=f^{\prime \prime \prime} / f^{\prime}-(3 / 2)\left(f^{\prime \prime} / f^{\prime}\right)^{2}>0$;

3. Functions $\psi \in \mathrm{E}(A, B)$ satisfy so-called a priori bounds:

For $x<0$ and $x>1$ :

$$
\begin{aligned}
& \frac{1-x}{1-\lambda \tilde{\lambda} x} \leq \psi(x) \leq \frac{1-x}{1+\tilde{\lambda} x}, \\
& \frac{1-x}{1-\lambda \tilde{\lambda} x} \leq \tilde{\psi}(x) \leq \frac{1-x}{1+\lambda x}
\end{aligned}
$$

and for $0<x<1$ :

$$
\begin{aligned}
& \frac{1-x}{1+\tilde{\lambda} x} \leq \psi(x) \leq \frac{1-x}{1-\lambda \tilde{\lambda} x}, \\
& \frac{1-x}{1+\lambda x} \leq \tilde{\psi}(x) \leq \frac{1-x}{1-\lambda \tilde{\lambda} x} .
\end{aligned}
$$

In $[20]$ we prove the following

Theorem. For each $\mu>0$ and for each $d>1$, there exists a solution pair $(\psi, \tilde{\psi})$ for $(2.10)$ with $\psi \in E\left(-\tilde{\lambda}^{-1},(\lambda \tilde{\lambda})^{-1}\right)$ and $\tilde{\psi} \in E\left(-\lambda^{-1},(\lambda \tilde{\lambda})^{-1}\right)$. 
From this it is straightforward to reverse the transformation above to show that (1.1) has a solution. See [20].

The following results are either explicitly stated in [20] or are simple consequences of the arguments therein.

Proposition 1. For a solution pair given by the theorem above we have

$$
\begin{aligned}
& \text { 1. } \lambda \leq z_{1} \text {, and } \tilde{\lambda} \leq \tilde{z}_{1} ; \\
& \text { 2. } \lambda \in\left[0, \mu^{-1 / d}\right] \text { and } \tilde{\lambda} \in\left[0, \mu^{1 / d}\right] ; \\
& \text { 3. } z_{1}, \tilde{z}_{1}, \tau, \tilde{\tau} \in(0,1) ; \\
& \text { 4. } 0<\lambda \tilde{\lambda}<1 ; \\
& \text { 5. } \tau \tilde{\tau}=(\lambda \tilde{\lambda})^{d} \leq 1 /(1+\lambda \tilde{\lambda}) ; \\
& \text { 6. } \psi \text { and } \tilde{\psi} \text { may be extended continuously to }-\tilde{\lambda}^{-1} \text { and }-\lambda^{-1} \text { respectively and satisfy } \\
& \quad \psi\left(-\tilde{\lambda}^{-1}\right)=\tilde{\tau}^{-1} \text { and } \tilde{\psi}\left(-\lambda^{-1}\right)=\tau^{-1} \text {. }
\end{aligned}
$$

We shall use these results in the proofs that follow.

\section{$3 \quad$ The limit $d \rightarrow 1$, fixed $\mu \neq 1$}

Without loss of generality we shall confine attention to the case $\mu>1$. The case $\mu<1$ merely corresponds to an interchange of $\lambda$ with $\tilde{\lambda}$. As in [20], it will be convenient to use the notation

$$
\nu=\mu^{1 / d}
$$

It will also be convenient to introduce the perturbation parameter $\varepsilon$ by writing

$$
d=1+\varepsilon
$$

Henceforth when we write the limit $d \rightarrow 1$ it is to be understood that the right-hand limit $d \rightarrow 1+$ is intended.

In this section we shall prove the following theorem, from which the theorem in Section 1 follows.

Theorem. Let $\mu>1$ be fixed. Then, for any family (not necessarily continuous) of solutions of equations (2.10) parametrised by $d>1$, we have that as $d \rightarrow 1+$

$$
\begin{aligned}
& \lambda=C \mu^{-1 /(2(d-1))}(1+o(1)) \\
& \tilde{\lambda}=(\sqrt{\mu}-1)(1+o(1)),
\end{aligned}
$$

where

$$
C=\frac{\mu^{(1+2 \sqrt{\mu}) /(4(\sqrt{\mu}-1))}}{e^{3}(\sqrt{\mu}-1)^{1 / 2}} .
$$

For the remainder of this paper we assume that we have a family of solutions of (2.10) as in the statement of the theorem.

We shall build up our results as a sequence of lemmas. 
Lemma 1. For $\psi \in E\left(-\tilde{\lambda}^{-1},(\lambda \tilde{\lambda})^{-1}\right), \tilde{\psi} \in E\left(-\lambda^{-1},(\lambda \tilde{\lambda})^{-1}\right)$, and with $\psi$ and $\tilde{\psi}$ having continuous extensions to $\tilde{\lambda}^{-1}$ and $-\lambda^{-1}$ respectively, and satisfying $\psi\left(-\tilde{\lambda}^{-1}\right)=\tilde{\tau}^{-1}, \tilde{\psi}\left(-\lambda^{-1}\right)=$ $\tau^{-1}$, we have

$$
\begin{array}{ll}
\psi(x) \leq \frac{1-x}{(\tilde{\tau}+\tilde{\lambda} \tilde{\tau}-\tilde{\lambda})(1-x)+(1-\tilde{\tau})(1+\tilde{\lambda})} & \text { for } \quad x \in\left(-\tilde{\lambda}^{-1}, 0\right), \\
\tilde{\psi}(x) \leq \frac{1-x}{(\tau+\lambda \tau-\lambda)(1-x)+(1-\tau)(1+\lambda)} \quad \text { for } \quad x \in\left(-\lambda^{-1}, 0\right) .
\end{array}
$$

Proof. Let $\theta(x)$ be the unique fractional linear transformation preserving 0 and 1 , and which maps $1+\tilde{\lambda}^{-1}$ to $\tilde{\tau}^{-1}$ :

$$
\theta(x)=\frac{x}{(\tilde{\tau}+\tilde{\lambda} \tilde{\tau}-\tilde{\lambda}) x+(1-\tilde{\tau})(1+\tilde{\lambda})} .
$$

Then $\theta^{-1} \circ \psi$ has positive Schwarzian derivative, intersects the line $1-x$ at the points $-\tilde{\lambda}^{-1}$, 0 , and 1 , and moreover

$$
\left(\theta^{-1} \circ \psi\right)^{\prime}(x) \rightarrow-\infty \quad \text { as } \quad x \rightarrow-\tilde{\lambda}^{-1}
$$

(This can be seen by differentiating the functional equations (2.11) and letting $x \rightarrow-\tilde{\lambda}^{-1}$, noting that $\theta^{-1}$ is an increasing fractional linear transformation, and, hence, has positive derivative.) We conclude that $\left(\theta^{-1} \circ \psi\right)(x)<1-x$ for $x$ slightly above $-\tilde{\lambda}^{-1}$ so that we have

$$
\left(\theta^{-1} \circ \psi\right)(x) \leq 1-x \quad \text { for } \quad x \in\left(-\tilde{\lambda}^{-1}, 0\right) .
$$

Since $\theta$ is increasing we deduce that

$$
\psi(x) \leq \theta(1-x)
$$

and hence the result.

The proof for $\tilde{\psi}$ is similar.

Lemma 2. $\lambda \tilde{\lambda} \rightarrow 0$ as $d \rightarrow 1$.

Proof. Suppose to the contrary that $\lambda \tilde{\lambda} \nrightarrow 0$ as $d \rightarrow 1$. Then there exists a sequence $\left(d_{i}\right)$ convergent to 1 such that the corresponding sequence $(\lambda \tilde{\lambda})$ is bounded away from 0 . We note, taking the limit $d \rightarrow 1$ of property 5 of Proposition 1 , that $(\lambda \tilde{\lambda})$ is also bounded away from 1. Since $\lambda \in\left[0, \nu^{-1}\right]$ and $\tilde{\lambda} \in[0, \nu]$ it follows that $\lambda \nrightarrow 0$ and $\tilde{\lambda} \not 0$ for this sequence. Now consider a subsequence for which both $\lambda$ and $\tilde{\lambda}$ converge, with limits $\lambda_{1}>0$ and $\tilde{\lambda}_{1}>0$ say. Then, for this subsequence, $\psi \in \mathrm{E}\left(-\tilde{\lambda}^{-1},(\lambda \tilde{\lambda})^{-1}\right)$ and $\tilde{\psi} \in \mathrm{E}\left(-\lambda^{-1},(\lambda \tilde{\lambda})^{-1}\right)$ will be contained in a compact space of anti-Herglotz functions. (In fact $\psi \in \mathrm{E}\left(-\tilde{b}^{-1}, c^{-1}\right)$ and $\tilde{\psi} \in \mathrm{E}\left(-b^{-1}, c^{-1}\right)$ where $b=\sup \lambda, \tilde{b}=\sup \tilde{\lambda}$ and $c=\sup \lambda \tilde{\lambda}$. $)$ We may therefore restrict further to obtain a convergent subsequence $(\psi, \tilde{\psi}) \rightarrow\left(\psi_{1}, \tilde{\psi}_{1}\right)$, and, since $\lambda \rightarrow \lambda_{1}$ and $\tilde{\lambda} \rightarrow \tilde{\lambda}_{1}$, we may extract a subsequence such that $(\psi, \tilde{\psi}) \rightarrow\left(\psi_{1}, \tilde{\psi}_{1}\right)$, where $\psi_{1} \in \mathrm{E}\left(-\tilde{\lambda}_{1}^{-1},\left(\lambda_{1} \tilde{\lambda}_{1}\right)^{-1}\right)$ and $\tilde{\psi}_{1} \in \mathrm{E}\left(-\lambda_{1}^{-1},\left(\lambda_{1} \tilde{\lambda}_{1}\right)^{-1}\right)$.

Furthermore we have that

$$
\psi_{1}(x)=\tilde{\tau}_{1}^{-1} \tilde{\psi}_{1}\left(\tilde{\phi}_{1}(x)\right), \quad \tilde{\psi}_{1}(x)=\tau_{1}^{-1} \psi_{1}\left(\phi_{1}(x)\right),
$$

where

$$
\phi_{1}(x)=\frac{\psi_{1}\left(-\lambda_{1} x\right)}{\psi_{1}\left(-\lambda_{1}\right)}, \quad \tilde{\phi}_{1}(x)=\frac{\tilde{\psi}_{1}\left(-\tilde{\lambda}_{1} x\right)}{\tilde{\psi}_{1}\left(-\tilde{\lambda}_{1}\right)}
$$


and

$$
\tau_{1}=\psi_{1}\left(\psi_{1}\left(-\lambda_{1}\right)^{-1}\right), \quad \tilde{\tau}_{1}=\tilde{\psi}_{1}\left(\tilde{\psi}_{1}\left(-\tilde{\lambda}_{1}\right)^{-1}\right)
$$

For clarity we now drop the subscript 1 . We note that Proposition 1 holds in the limit $d \rightarrow 1$. Now, we have $z_{1}=\psi(-\lambda)^{-1}$, and, by Lemma 1 ,

$$
\psi(-\lambda) \leq \frac{1+\lambda}{(\tilde{\tau}+\tilde{\lambda} \tilde{\tau}-\tilde{\lambda})(1+\lambda)+(1-\tilde{\tau})(1+\tilde{\lambda})},
$$

and so

$$
z_{1} \geq \frac{1+\lambda \tilde{\tau}+\lambda \tilde{\lambda}(\tilde{\tau}-1)}{1+\lambda}
$$

Thus the a priori bounds show that $\tau=\psi\left(z_{1}\right)$ satisfies

$$
\begin{aligned}
\tau \leq \frac{1-z_{1}}{1-\lambda \tilde{\lambda} z_{1}} & \leq \frac{1-(1+\lambda \tilde{\tau}+\lambda \tilde{\lambda}(\tilde{\tau}-1)) /(1+\lambda)}{1-\lambda \tilde{\lambda}(1+\lambda \tilde{\tau}+\lambda \tilde{\lambda}(\tilde{\tau}-1)) /(1+\lambda)} \\
& =\frac{\lambda(1-\tilde{\tau})(1+\tilde{\lambda})}{(1+\lambda)(1-\lambda \tilde{\lambda}(1+\lambda \tilde{\tau}+\lambda \tilde{\lambda}(\tilde{\tau}-1)) /(1+\lambda))} \\
& \leq \frac{\lambda(1-\tilde{\tau})(1+\tilde{\lambda})}{(1+\lambda)(1-\lambda \tilde{\lambda})}
\end{aligned}
$$

where in the last step we have used the fact that

$$
0 \leq \frac{1+\lambda \tilde{\tau}+\lambda \tilde{\lambda}(\tilde{\tau}-1)}{1+\lambda} \leq 1
$$

A similar argument shows that

$$
\tilde{\tau} \leq \frac{\tilde{\lambda}(1-\tau)(1+\lambda)}{(1+\tilde{\lambda})(1-\lambda \tilde{\lambda})}
$$

and thus (remembering we are at the limit $d=1$ )

$$
\lambda \tilde{\lambda}=(\lambda \tilde{\lambda})^{d}=\tau \tilde{\tau} \leq \frac{\lambda \tilde{\lambda}(1-\tau)(1-\tilde{\tau})}{(1-\lambda \tilde{\lambda})^{2}},
$$

which gives the contradiction

$$
1 \leq \frac{(1-\tau)(1-\tilde{\tau})}{(1-\tau \tilde{\tau})^{2}}<1
$$

where, for the final inequality, we have used $0<\tau, \tilde{\tau}<1$ (property 3 of Proposition 1 ) and $0<\tau \tilde{\tau}<1$, which follows from property 5 of Proposition 1, taking $d \rightarrow 1$.

Lemma 3. $\lambda \rightarrow 0$ as $d \rightarrow 1$.

Proof. The a priori bounds give

$$
\frac{1+\lambda}{1+\lambda^{2} \tilde{\lambda}} \leq \psi(-\lambda) \leq \frac{1+\lambda}{1-\lambda \tilde{\lambda}}
$$


Suppose $\lambda \nrightarrow 0$ as $d \rightarrow 1$ (and thus $\tilde{\lambda} \rightarrow 0$ by Lemma 2 ). Then (as earlier) there is a sequence $\left(d_{i}\right)$ convergent to 1 such that the corresponding sequence $(\lambda)$ converges with limit $r \in(h, 1]$ where $h>0$. The a priori bounds act as a sandwich and we deduce from Lemma 2 (for this sequence) that $\psi(-\lambda) \rightarrow 1+r$. Hence $z_{1}=\psi(-\lambda)^{-1 / d} \rightarrow(1+r)^{-1}$.

Using the a priori bounds we have

$$
\frac{1-z_{1}}{1+\tilde{\lambda} z_{1}} \leq \psi\left(z_{1}\right) \leq \frac{1-z_{1}}{1-\lambda \tilde{\lambda} z_{1}}
$$

which gives $\tau=\psi\left(z_{1}\right) \rightarrow 1-(1+r)^{-1}=r /(1+r)$.

Now from (2.14) we have

$$
\lambda^{d}=\frac{z_{1}^{d} \tau}{\mu \tilde{z}_{1}^{d}}
$$

and so we have

$$
\tilde{z}_{1}=\tilde{\psi}(-\tilde{\lambda})^{-1 / d} \rightarrow \frac{1}{\mu(1+r)^{2}}<1
$$

But $\tilde{\lambda} \rightarrow 0$, so from the a priori bounds $\tilde{\psi}(-\tilde{\lambda})^{-1 / d} \rightarrow 1$ which is a contradiction. Hence $\lambda \rightarrow 0$ as $d \rightarrow 1$.

Lemma $4 . \tilde{\lambda} \rightarrow \sqrt{\mu}-1$ as $d \rightarrow 1$.

Proof. The a priori bounds give

$$
\frac{1}{1+\lambda \tilde{\lambda}^{2}} \leq \frac{\tilde{\psi}(-\tilde{\lambda})}{1+\tilde{\lambda}} \leq \frac{1}{1-\lambda \tilde{\lambda}} .
$$

Since $\tilde{\lambda}$ is bounded, by Lemma 2 we have

$$
\lim _{d \rightarrow 1} \frac{\tilde{\psi}(-\tilde{\lambda})}{1+\tilde{\lambda}}=1
$$

i.e., $\tilde{\psi}(-\tilde{\lambda}) \sim 1+\tilde{\lambda}$ as $d \rightarrow 1$. Hence

$$
\tilde{z}_{1}=\tilde{\psi}(-\tilde{\lambda})^{-1 / d} \sim \frac{1}{1+\tilde{\lambda}} \quad \text { as } d \rightarrow 1
$$

Thus, using the a priori bounds, $\tilde{\tau}=\tilde{\psi}\left(\tilde{z}_{1}\right)$ satisfies

$$
\frac{1-\tilde{z}_{1}}{1+\lambda \tilde{z}_{1}} \leq \tilde{\tau} \leq \frac{1-\tilde{z}_{1}}{1-\lambda \tilde{\lambda} \tilde{z}_{1}}
$$

and so

$$
\tilde{\tau} \sim \frac{\tilde{\lambda}}{1+\tilde{\lambda}}
$$

Now, using the a priori bounds again, $\psi(-\lambda)$ satisfies

$$
\frac{1+\lambda}{1+\lambda^{2} \tilde{\lambda}} \leq \psi(-\lambda) \leq \frac{1+\lambda}{1-\lambda \tilde{\lambda}}
$$


and so since $\lambda \rightarrow 0$ (by Lemma 3 ) we have $\psi(-\lambda) \rightarrow 1$ and $z_{1}=\psi(-\lambda)^{-1 / d} \rightarrow 1$ as $d \rightarrow 1$. Now in (2.14)

$$
\tilde{\lambda}^{d}=\frac{\mu \tilde{\tau} \psi(-\lambda)}{\tilde{\psi}(-\tilde{\lambda})}
$$

we take the limit $d \rightarrow 1$ to get

$$
\tilde{\lambda} \sim \frac{\mu \tilde{\lambda}}{(1+\tilde{\lambda})(1+\tilde{\lambda})}
$$

which gives $\tilde{\lambda} \rightarrow \sqrt{\mu}-1$ as $d \rightarrow 1$ as desired.

Lemma 5. For $x \in\left(-\lambda^{-1},(\lambda \tilde{\lambda})^{-1}\right)$ we have $\tilde{\psi}(x) \rightarrow 1-x$ and for $x \in\left(-\tilde{\lambda}^{-1},(\lambda \tilde{\lambda})^{-1}\right)$ we have $\psi(x) \rightarrow 1-x$ as $d \rightarrow 1$.

Proof. The a priori bounds clearly show that $\tilde{\psi}(x) \rightarrow 1-x$ as $d \rightarrow 1$. The behaviour of $\psi$ is not so immediate.

We have (2.11)

$$
\psi(x)=\tilde{\tau}^{-1} \tilde{\psi}(\tilde{\phi}(x))
$$

Now as $d \rightarrow 1$ the behaviour of $\tilde{z}_{1}(3.28)$ and $\tilde{\psi}$ show

$$
\tilde{\phi}(x)=\tilde{z}_{1} \tilde{\psi}(-\tilde{\lambda} x)^{1 / d}=\frac{1+\tilde{\lambda} x}{1+\tilde{\lambda}}+o(1) .
$$

Also (using (3.30) and Lemma 4)

$$
\tilde{\tau}=\frac{\sqrt{\mu}-1}{\sqrt{\mu}}+o(1)
$$

hence

$$
\begin{aligned}
\psi(x) & =\left(\frac{1+\tilde{\lambda}}{\tilde{\lambda}}+o(1)\right)\left(1-\left(\frac{1+\tilde{\lambda} x}{1+\tilde{\lambda}}+o(1)\right)+o(1)\right) \\
& =\frac{1+\tilde{\lambda}}{\tilde{\lambda}}\left(\frac{1+\tilde{\lambda}-(1+\tilde{\lambda} x)}{1+\tilde{\lambda}}\right)+o(1) \\
& =1-x+o(1),
\end{aligned}
$$

Thus $\psi(x) \rightarrow 1-x$ as required.

Lemma 6. For $x \in\left(-\lambda^{-1},(\lambda \tilde{\lambda})^{-1}\right), \tilde{\psi}^{\prime}(x) \rightarrow-1$ as $d \rightarrow 1$.

Proof. Recall that $\tilde{\psi} \in \mathrm{AH}\left(-\lambda^{-1},(\lambda \tilde{\lambda})^{-1}\right)$ and satisfies $\tilde{\psi}(0)=1, \tilde{\psi}(1)=0$.

Suppose that, for a fixed $x, \psi^{\prime}(x) \nrightarrow-1$. Then there is a sequence of $d$ 's such that $\psi^{\prime}(x) \nrightarrow \rightarrow$ -1 . Restricting to this sequence we have a sequence of $\psi$ 's converging to $1-x$ in $\mathrm{AH}(A, B)$ with $A<0<1<B$. For a convergent subsequence we then have $\tilde{\psi}^{\prime}(x) \rightarrow-1$ which is a contradiction.

Lemma 7. For $x \in\left(-\tilde{\lambda}^{-1},(\lambda \tilde{\lambda})^{-1}\right), \psi^{\prime}(x) \rightarrow-1$ as $d \rightarrow 1$. 
Proof. We differentiate (2.11)

$$
\psi(x)=\tilde{\tau}^{-1} \tilde{\psi}\left(\tilde{z}_{1} \tilde{\psi}(-\tilde{\lambda} x)^{1 / d}\right)
$$

to get

$$
\psi^{\prime}(x)=\tilde{\tau}^{-1} \tilde{\psi}^{\prime}\left(\tilde{z}_{1} \tilde{\psi}(-\tilde{\lambda} x)^{1 / d}\right) \frac{\tilde{z}_{1}}{d} \tilde{\psi}(-\tilde{\lambda})^{1 / d-1} \tilde{\psi}^{\prime}(-\tilde{\lambda} x)(-\tilde{\lambda}) .
$$

Taking the limit $d \rightarrow 1$, using the fact that $\tilde{\tau} \sim \tilde{\lambda} /(1+\tilde{\lambda})$, we have $\psi^{\prime}(x) \rightarrow-1$.

(We have also used $\tilde{z}_{1} \rightarrow 1 / \sqrt{\mu}, d \rightarrow 1$, and $\tilde{\psi}^{\prime} \rightarrow-1$.)

Lemma 8. $\tau \sim \lambda$ as $d \rightarrow 1$.

Proof. Recall that $\tau=\psi\left(z_{1}\right)=\psi\left(\psi(-\lambda)^{-1 / d}\right)$.

Consider the function $f(y)=\psi\left(\psi(-y)^{-1 / d}\right)$, which is Herglotz and analytic at 0 with $f(0)=$ 0 . We have

$$
f^{\prime}(y)=\psi^{\prime}\left(\psi(-y)^{-1 / d}\right) \frac{-1}{d} \psi(-y)^{-1 / d-1} \psi^{\prime}(-y)(-1)
$$

so

$$
f^{\prime}(0)=\frac{\psi^{\prime}(1) \psi^{\prime}(0)}{d}
$$

Expanding in a Taylor series

$$
f(y)=\frac{\psi^{\prime}(1) \psi^{\prime}(0)}{d} y+o(y)
$$

Now

$$
\tau=f(\lambda)=\frac{\psi^{\prime}(1) \psi^{\prime}(0)}{d} \lambda+o(\lambda)
$$

hence

$$
\frac{\tau}{\lambda} \rightarrow 1
$$

using the fact that $\psi^{\prime}(0), \psi^{\prime}(1) \rightarrow-1$ as $d \rightarrow 1$.

Lemma 9. We have $\lambda^{\varepsilon} \rightarrow 1 / \sqrt{\mu}$ as $d \rightarrow 1$.

Proof. From previous lemmas we have $\lambda \rightarrow 0, \tau=\lambda+o(\lambda), z_{1}=\psi(-\lambda)^{-1 / d}=1+o(1)$, and $\tilde{z}_{1}=1 / \sqrt{\mu}+o(1)$. Hence $(2.14)$

$$
\lambda^{d}=\frac{\tau z_{1}^{d}}{\mu \tilde{z}_{1}^{d}}=\frac{(\lambda+o(\lambda))(1+o(1))}{\mu(1 / \sqrt{\mu}+o(1))}
$$

and so

$$
\lambda^{\varepsilon}=\lambda^{d-1}=\frac{1}{\sqrt{\mu}}+o(1) .
$$

Corollary 1. $\lambda=o\left(\varepsilon^{\alpha}\right)$ for every $\alpha>0$.

Corollary 2. For fixed $x \in\left(-\lambda^{-1},(\lambda \tilde{\lambda})^{-1}\right), \tilde{\psi}(x)=1-x+o\left(\varepsilon^{\alpha}\right)$ for every $\alpha>0$.

Proof. This follows immediately from Corollary 1 and the a priori bounds. 


\section{Calculations to $O(\varepsilon)$ as $d \rightarrow 1$}

In this section we explore further the limiting behaviours of the previous section. For $\tilde{\lambda}$ we shall calculate the first order correction to Lemma 4. Lemma 9 give an indication of the behaviour of $\lambda$. We shall make a more precise statement here.

Firstly we shall deal with $\tilde{\lambda}$. We expand the terms in the functional equation for $\psi(2.10)$

$$
\psi(x)=\frac{\mu \psi(-\lambda)}{\tilde{\lambda}^{d} \tilde{\psi}(-\tilde{\lambda})} \tilde{\psi}\left(\tilde{z}_{1} \tilde{\psi}(-\tilde{\lambda} x)^{1 / d}\right) .
$$

as series in $\varepsilon$. (Recall our notation that $d=1+\varepsilon$.) Firstly

$$
\begin{aligned}
\tilde{\psi}(-\tilde{\lambda} x) & =1+\tilde{\lambda} x+O(\lambda), \\
\tilde{\psi}(-\tilde{\lambda}) & =1+\tilde{\lambda}+O(\lambda)
\end{aligned}
$$

SO

$$
\tilde{\phi}(x)=\frac{\tilde{\psi}(-\tilde{\lambda} x)^{1 / d}}{\tilde{\psi}(-\tilde{\lambda})^{1 / d}}=\left(\frac{1+\tilde{\lambda} x}{1+\tilde{\lambda}}\right)^{1 / d}+O(\lambda) .
$$

Now, since $X^{1 /(1+\varepsilon)}=X-\varepsilon X \log X+O\left(\varepsilon^{2}\right)$, this is

$$
\tilde{\phi}(x)=\left(\frac{1+\tilde{\lambda} x}{1+\tilde{\lambda}}\right)\left(1-\varepsilon \log \left(\frac{1+\tilde{\lambda} x}{1+\tilde{\lambda}}\right)+O\left(\varepsilon^{2}\right)\right)+O(\lambda) .
$$

Hence

$$
\begin{aligned}
\tilde{\psi}(\tilde{\phi}(x)) & =1-\tilde{\phi}(x)+O(\lambda) \\
& =1-\left(\frac{1+\tilde{\lambda} x}{1+\tilde{\lambda}}\right)\left(1-\varepsilon \log \left(\frac{1+\tilde{\lambda} x}{1+\tilde{\lambda}}\right)+O\left(\varepsilon^{2}\right)\right)+O(\lambda) \\
& =\frac{\tilde{\lambda}(1-x)}{1+\tilde{\lambda}}+\varepsilon\left(\frac{1+\tilde{\lambda} x}{1+\tilde{\lambda}}\right) \log \left(\frac{1+\tilde{\lambda} x}{1+\tilde{\lambda}}\right)+O\left(\varepsilon^{2}\right) .
\end{aligned}
$$

(We can absorb the term $O(\lambda)$ into the term $O\left(\varepsilon^{2}\right)$ since $\lambda=o\left(\varepsilon^{\alpha}\right)$ for all $\alpha>0$.)

Thus, using (4.1),

$$
\psi(x)=\frac{\mu \psi(-\lambda)}{\tilde{\lambda}^{d}(1+\tilde{\lambda}+O(\lambda))}\left(\frac{\tilde{\lambda}(1-x)}{1+\tilde{\lambda}}+\varepsilon\left(\frac{1+\tilde{\lambda} x}{1+\tilde{\lambda}}\right) \log \left(\frac{1+\tilde{\lambda} x}{1+\tilde{\lambda}}\right)+O\left(\varepsilon^{2}\right)\right) .
$$

Now the a priori bounds for $\psi$ give

$$
\frac{1+\lambda}{1+\lambda^{2} \tilde{\lambda}} \leq \psi(-\lambda) \leq \frac{1+\lambda}{1-\lambda \tilde{\lambda}}
$$

from which we see that

$$
\psi(-\lambda)=1+O(\lambda)
$$

and so

$$
\psi(x)=\frac{\mu}{\tilde{\lambda}^{d}(1+\tilde{\lambda})}\left(\frac{\tilde{\lambda}(1-x)}{1+\tilde{\lambda}}+\varepsilon\left(\frac{1+\tilde{\lambda} x}{1+\tilde{\lambda}}\right) \log \left(\frac{1+\tilde{\lambda} x}{1+\tilde{\lambda}}\right)+O\left(\varepsilon^{2}\right)\right) .
$$


Evaluating at $x=0$ we obtain

$$
1=\frac{\mu}{\tilde{\lambda}^{d-1}(1+\tilde{\lambda})^{2}}+\frac{\varepsilon \mu}{\tilde{\lambda}^{d}(1+\tilde{\lambda})^{2}} \log \frac{1}{(1+\tilde{\lambda})}+O\left(\varepsilon^{2}\right)
$$

which is an equation for $\tilde{\lambda}$ to order $\varepsilon$. The solution of this regular perturbation problem is $\tilde{\lambda}=\sqrt{\mu}-1+O(\varepsilon)$. Writing $\tilde{\lambda}=\sqrt{\mu}-1+\varepsilon \tilde{\lambda}_{1}+O\left(\varepsilon^{2}\right)$ we have

$$
\begin{aligned}
\tilde{\lambda}^{d} & =\sqrt{\mu}-1+\varepsilon\left(\tilde{\lambda}_{1}+(\sqrt{\mu}-1) \log (\sqrt{\mu}-1)\right)+O\left(\varepsilon^{2}\right), \\
(1+\tilde{\lambda})^{2} & =\mu+2 \varepsilon \sqrt{\mu} \tilde{\lambda}_{1}+O\left(\varepsilon^{2}\right), \\
\log \frac{1}{(1+\tilde{\lambda})} & =-\log \sqrt{\mu}-\varepsilon \frac{\tilde{\lambda}_{1}}{\sqrt{\mu}}+O\left(\varepsilon^{2}\right)=-\log \sqrt{\mu}+O(\varepsilon) .
\end{aligned}
$$

Thus our equation (4.13)

$$
\tilde{\lambda}^{d}(1+\tilde{\lambda})^{2}=\mu \tilde{\lambda}+\varepsilon \mu \log \frac{1}{(1+\tilde{\lambda})}+O\left(\varepsilon^{2}\right)
$$

becomes

$$
\begin{aligned}
\left(\sqrt{\mu}-1+\varepsilon\left(\tilde{\lambda}_{1}+(\sqrt{\mu}-1) \log (\sqrt{\mu}-1)\right)+O\left(\varepsilon^{2}\right)\right) \\
\times\left(\mu+2 \varepsilon \sqrt{\mu} \tilde{\lambda}_{1}+O\left(\varepsilon^{2}\right)\right) \\
=\mu\left(\sqrt{\mu}-1+\varepsilon \tilde{\lambda}_{1}+O\left(\varepsilon^{2}\right)+\varepsilon(-\log \sqrt{\mu}+O(\varepsilon))\right),
\end{aligned}
$$

which can readily be solved to give

$$
\tilde{\lambda}_{1}=\frac{-\sqrt{\mu}}{2(\sqrt{\mu}-1)} \log \left(\sqrt{\mu}(\sqrt{\mu}-1)^{\sqrt{\mu}-1}\right) .
$$

From now on we assume that $\tilde{\lambda}_{1}$ is given by this expression and

$$
\tilde{\lambda}=\sqrt{\mu}-1+\varepsilon \tilde{\lambda}_{1}+O\left(\varepsilon^{2}\right) .
$$

We now consider $\lambda$. We have $((2.14))$

$$
\lambda^{d}=\frac{\tau \tilde{\psi}(-\tilde{\lambda})}{\mu \psi(-\lambda)} .
$$

For the term $\tilde{\psi}(-\tilde{\lambda})$ we use (4.3) and (4.20) to write

$$
\tilde{\psi}(-\tilde{\lambda})=\sqrt{\mu}+\varepsilon \tilde{\lambda}_{1}+O\left(\varepsilon^{2}\right) .
$$

For the term $\psi(-\lambda)$ in the denominator it will suffice to write $((4.11))$

$$
\psi(-\lambda)=1+O(\lambda)=1+O\left(\varepsilon^{2}\right),
$$

but we must be more careful with $\tau=\psi\left(z_{1}\right) \sim \lambda$.

By (4.9), we can write

$$
\psi(x)=1-x+\varepsilon \psi_{1}(x)+o(\varepsilon) \psi_{2}(x),
$$


where $\psi_{1}$ and $\psi_{2}$ are analytic on $\left(-\tilde{\lambda}^{-1},(\lambda \tilde{\lambda})^{-1}\right)$ and where $\psi_{1}(0)=0, \psi_{1}(1)=0$, and $\psi_{2}(0)=0, \psi_{2}(1)=0$. Thus

$$
\psi(-\lambda)=1+\lambda+\varepsilon \psi_{1}(-\lambda)+o(\varepsilon \lambda)
$$

with

$$
\psi_{1}(-\lambda)=\psi_{1}^{\prime}(0)(-\lambda)+O\left(\lambda^{2}\right)
$$

Hence

$$
\psi(-\lambda)=1+\lambda-\varepsilon \lambda \psi_{1}^{\prime}(0)+o(\varepsilon \lambda) .
$$

Now $z_{1}=\psi(-\lambda)^{-1 / d}$ and so

$$
\begin{aligned}
\log z_{1} & =\frac{-1}{1+\varepsilon} \log \psi(-\lambda) \\
& =\frac{-1}{1+\varepsilon} \log \left(1+\lambda-\varepsilon \lambda \psi_{1}^{\prime}(0)+o(\varepsilon \lambda)\right) \\
& =-\lambda+\varepsilon \lambda \psi_{1}^{\prime}(0)+\varepsilon \lambda+o(\varepsilon \lambda)
\end{aligned}
$$

hence

$$
z_{1}=1-\lambda+\varepsilon \lambda\left(1+\psi_{1}^{\prime}(0)\right)+o(\varepsilon \lambda)
$$

and thus

$$
\begin{aligned}
\tau=\psi\left(z_{1}\right)=1- & z_{1}+\varepsilon \psi_{1}\left(z_{1}\right)+o(\varepsilon) \psi_{2}\left(z_{1}\right) \\
=1 & \left(1-\lambda+\varepsilon \lambda\left(1+\psi_{1}^{\prime}(0)\right)+o(\varepsilon \lambda)\right) \\
& +\varepsilon \psi_{1}\left(1-\lambda+\varepsilon \lambda\left(1+\psi_{1}^{\prime}(0)\right)+o(\varepsilon \lambda)\right)+o(\varepsilon \lambda) \\
=\lambda & +\varepsilon \lambda\left(-1-\psi_{1}^{\prime}(0)-\psi_{1}^{\prime}(1)\right)+o(\varepsilon \lambda) .
\end{aligned}
$$

So (4.21) becomes

$$
\left.\lambda^{d}=\left(\sqrt{\mu}+\varepsilon \tilde{\lambda}_{1}+O\left(\varepsilon^{2}\right)\right)\left(\lambda+\varepsilon \lambda\left(-1-\psi_{1}^{\prime}(0)-\psi_{1}^{\prime}(1)\right)+o(\varepsilon \lambda)\right)\right) /\left(\mu\left(1+O\left(\varepsilon^{2}\right)\right)\right),
$$

and thus

$$
\begin{aligned}
\lambda^{\varepsilon} & =\frac{1}{\mu}\left(\sqrt{\mu}+\varepsilon \tilde{\lambda}_{1}+O\left(\varepsilon^{2}\right)\right)\left(1+\varepsilon\left(-1-\psi_{1}^{\prime}(0)-\psi_{1}^{\prime}(1)\right)+o(\varepsilon)\right) \\
& =\frac{1}{\sqrt{\mu}}\left(1+\varepsilon\left(-1-\psi_{1}^{\prime}(0)-\psi_{1}^{\prime}(1)+\frac{\tilde{\lambda}_{1}}{\sqrt{\mu}}\right)\right)+o(\varepsilon)
\end{aligned}
$$

and so

$$
\lambda=\left(\lambda^{\varepsilon}\right)^{1 / \varepsilon} \sim\left(\frac{1}{\sqrt{\mu}}\right)^{1 / \varepsilon} \exp \left(-1-\psi_{1}^{\prime}(0)-\psi_{1}^{\prime}(1)+\frac{\tilde{\lambda}_{1}}{\sqrt{\mu}}\right) .
$$

We now calculate the terms in the exponential explicitly. Note that

$$
\varepsilon \psi_{1}^{\prime}(x)=\psi^{\prime}(x)+1+o(\varepsilon) .
$$

Now (4.9) becomes

$$
\psi(x)=\frac{\mu \tilde{\lambda}(1-x)}{\tilde{\lambda}^{d}(1+\tilde{\lambda})^{2}}+\frac{\varepsilon \mu}{\tilde{\lambda}^{d}(1+\tilde{\lambda})}\left(\frac{1+\tilde{\lambda} x}{1+\tilde{\lambda}}\right) \log \left(\frac{1+\tilde{\lambda} x}{1+\tilde{\lambda}}\right)+o(\varepsilon)
$$


Differentiating we have

$$
\psi^{\prime}(x)=\frac{-\mu \tilde{\lambda}}{\tilde{\lambda}^{d}(1+\tilde{\lambda})^{2}}+\frac{\varepsilon \mu}{\tilde{\lambda}^{d-1}(1+\tilde{\lambda})^{2}}\left(1+\log \left(\frac{1+\tilde{\lambda} x}{1+\tilde{\lambda}}\right)\right)+o(\varepsilon),
$$

SO

$$
\begin{aligned}
& \psi^{\prime}(0)+1=1-\frac{\mu}{\tilde{\lambda}^{d-1}(1+\tilde{\lambda})^{2}}+\frac{\varepsilon \mu}{\tilde{\lambda}^{d-1}(1+\tilde{\lambda})^{2}}(1-\log (1+\tilde{\lambda}))+o(\varepsilon), \\
& \psi^{\prime}(1)+1=1-\frac{\mu}{\tilde{\lambda}^{d-1}(1+\tilde{\lambda})^{2}}+\frac{\varepsilon \mu}{\tilde{\lambda}^{d-1}(1+\tilde{\lambda})^{2}}+o(\varepsilon) .
\end{aligned}
$$

These can now be expanded to order $\varepsilon$ using (4.20). Straightforward calculations reveal

$$
\begin{aligned}
& \psi^{\prime}(0)+1=\varepsilon\left(1+\frac{2 \tilde{\lambda}_{1}}{\sqrt{\mu}}+\log (\sqrt{\mu}-1)-\log \sqrt{\mu}\right)+o(\varepsilon), \\
& \psi^{\prime}(1)+1=\varepsilon\left(1+\frac{2 \tilde{\lambda}_{1}}{\sqrt{\mu}}+\log (\sqrt{\mu}-1)\right)+o(\varepsilon),
\end{aligned}
$$

with $\tilde{\lambda}_{1}$ given by equation (4.19). Equation (4.39) now gives

$$
\begin{aligned}
& \psi_{1}^{\prime}(0)=1+\frac{2 \tilde{\lambda}_{1}}{\sqrt{\mu}}+\log (\sqrt{\mu}-1)-\log \sqrt{\mu}+O(\varepsilon), \\
& \psi_{1}^{\prime}(1)=1+\frac{2 \tilde{\lambda}_{1}}{\sqrt{\mu}}+\log (\sqrt{\mu}-1)+O(\varepsilon)
\end{aligned}
$$

so that (4.38) becomes

$$
\begin{aligned}
\lambda & \sim\left(\frac{1}{\sqrt{\mu}}\right)^{1 / \varepsilon} \exp \left(-3-\frac{3 \tilde{\lambda}_{1}}{\sqrt{\mu}}-2 \log (\sqrt{\mu}-1)+\log \sqrt{\mu}\right) \\
& =\left(\frac{1}{\sqrt{\mu}}\right)^{1 / \varepsilon} \frac{\mu^{(1+2 \sqrt{\mu}) /(4(\sqrt{\mu}-1))}}{e^{3}(\sqrt{\mu}-1)^{1 / 2}} .
\end{aligned}
$$

This completes the proof.

\section{Discussion}

In this paper we have successfully applied Herglotz function techniques to explore the asymptotics of period doubling in asymmetric unimodal maps in the limit degree $d \rightarrow 1$. Our results show that the symmetric $(\mu=1)$ and asymmetric cases have very different asymptotics.

It would certainly be of interest to explore other limits in the $(\mu, d)$-parameter space, for example the $d \rightarrow \infty$ limit, for fixed $\mu$.

In the symmetric case, the asymptotics as $d \rightarrow \infty$ are somewhat intriguing. Although $\lambda \rightarrow 1$ as $d \rightarrow \infty$, the approach is nontrivial. Using a variety of techniques, various numerical calculations have been performed $[12,25,26]$, the most accurate being those recently obtained by Briggs et al [1], who find a solution of the $d=\infty$ equations with $\tau_{\infty}=0.0333810598( \pm 5)$, where $\tau_{d}=\lambda^{d}$.

Using computer-assisted techniques, Eckmann and Wittwer [8] show there exists a solution with

$$
\tau_{\infty} \in[0.0333808,0.0333813]
$$


This (and other bounds obtained in [8]) has been used by Eckmann and Epstein [7] to obtain bounds on the unstable eigenvalue, $\delta_{\infty}$, for period doubling at $d=\infty$. They prove that

$$
\delta_{\infty} \in[29.5128,29.957112] \text {. }
$$

(In [1], Briggs et al estimate this as 29.576303( \pm 1$)$.) In [9] Epstein shows analytically that a solution of the $d=\infty$ equation exists with

$$
\tau_{\infty} \in[1 / 128,1 / 5]
$$

For the asymmetric case we expect a scenario somewhat similar to that of Eckmann-Wittwer [8]. We hope to study to this limit using Herglotz function techniques in the near future.

It would also be interesting to consider a different asymptotic regime, namely that for large $\mu$. This may give us some insight into the situation of asymmetric maps with different left and right degrees at the critical point. In this case numerical results $[14,3]$ indicate a loss of geometric scaling. Maps of this type were also used in the analysis of experiments on forced nonlinear oscillators [21].

A similar scenario is expected in the case of quasiperiodic orbits in circle maps. Indeed, the asymmetric case has already been considered in $[17,27]$. See also [18] and [16]. What is needed however is a proof of the existence (and knowledge of properties of) a universal period-two point, from which we expect asymptotic behaviour may be calculated. It seems likely that the Herglotz function approach will be fruitful here.

Asymptotics for the limit $d \rightarrow 1$ (always in the symmetric case) have been previously studied by several groups: Jonker and Rand [15], Ostlund et al [23], Shraiman [24], Dixon and Kenny [5]. The $d \rightarrow \infty$ limit has also been considered: Delbourgo and Kenny [4], Hu et al [13], Briggs et al [1], Dixon et al [6].

The asymptotics of scaling on the boundary of golden mean Siegel discs has been studied numerically by Osbaldestin [22]. Although the phenomenology seems identical to that in circle maps, there is no sight yet of a means of tackling this problem analytically. 


\section{References}

[1] K. M. Briggs, T. W. Dixon, and G. Szekeres, Analytic solutions of the CvitanovićFeigenbaum and Feigenbaum-Kadanoff-Shenker equations, Int. J. Bifurcation and Chaos 8 (1998), 347-357.

[2] P. Collet, J.-P. Eckmann, and O. E. Lanford, Universal properties of maps of an interval, Commun. Math. Phys. 76 (1980), 211-254.

[3] M. C. de Sousa Vieira, Influence of asymmetries on N-tupling sequences, Phys. Lett. A 143 (1990), 279-282.

[4] R. Delbourgo and B. G. Kenny, Relations between universal scaling constants for the circle map near the golden mean, J. Math. Phys. 32 (1991), 1045-1051.

[5] T. W. Dixon and B. G. Kenny, Transition to criticality in circle maps at the golden mean, Preprint, 1998.

[6] T. W. Dixon, B. G. Kenny, and K. M. Briggs, On the universality of singular circle maps, Phys. Lett. A 231 (1997), 359-366.

[7] J.-P. Eckmann and H. Epstein, Bounds on the unstable eigenvalue for period-doubling, Commun. Math. Phys. 128 (1990), 427-435.

[8] J.-P. Eckmann and P. Wittwer, Computer Methods and Borel Summability Applied to Feigenbaum's Equation, Lecture Notes in Physics, vol. 227, Springer-Verlag, Berlin, 1985 .

[9] H. Epstein, New proofs of the existence of the Feigenbaum functions, Commun. Math. Phys. 106 (1986), 395-426.

[10] _ Fixed points of composition operators, Nonlinear Evolution and Chaotic Phenomena (G. Gallavotti and P. Zweifel, eds.), Plenum, New York, 1988, pp. 71-100.

[11]_ Fixed points of composition operators II, Nonlinearity 2 (1989), 305-310.

[12] M. Feingold, D. L. Gonzalez, M. O. Magnasco, and O. Piro, On the universality class dependence of period doubling indices, Phys. Lett. A 156 (1991), 272-276.

[13] B. Hu, A. Valinia, and O. Piro, Universality and asymptotic limits of the scaling exponents in circle maps, Phys. Lett. A 144 (1990), 7-10.

[14] R. V. Jensen and L. K. H. Ma, Nonuniversal behavior of asymmetric unimodal maps, Phys. Rev. A 31 (1985), 3993-3995.

[15] L. Jonker and D. A. Rand, Universal properties of maps of the circle with $\varepsilon$-singularities, Commun. Math. Phys. 90 (1983), 273-292.

[16] K. M. Khanin and E. B. Vul, Circle homeomorphisms with weak discontinuities, Adv. Soviet Math. 3 (1991), 57-98.

[17] D. V. Lyubimov, A. S. Pikovsky, and M. A. Zaks, Universalities and scaling at the transition to chaos through homoclinic bifurcations, Renormalization Group (D. V. Shirkov, D. I. Kazakov, and A. A. Vladimirov, eds.), World Scientific, Singapore, 1986, pp. 278 289. 
[18] B. D. Mestel and A. H. Osbaldestin, Renormalisation in implicit complex maps, Physica D 39 (1989), 149-162.

[19] _ Feigenbaum theory for unimodal maps with asymmetric critical point, J. Phys. A 31 (1998), 3287-3296.

[20] _ Feigenbaum theory for unimodal maps with asymmetric critical point: rigorous results, Commun. Math. Phys. 197 (1998), 211-228.

[21] M. Octavio, A. DaCosta, and J. Aponte, Nonuniversality and metric properties of a forced nonlinear oscillator, Phys. Rev. A 34 (1986), 1512-1515.

[22] A. H. Osbaldestin, Siegel disc singularity spectra, J. Phys. A 25 (1992), 1169-1175.

[23] S. Ostlund, D. A. Rand, J. Sethna, and E. D. Siggia, Universal properties of the transition from quasi-periodicity to chaos in dissipative systems, Physica D 8 (1983), 303-342.

[24] B. I. Shraiman, Transition from quasiperiodicity to chaos: a perturbative renormalization-group approach, Phys. Rev. A 29 (1984), 3464-3466.

[25] C. J. Thompson and J. B. McGuire, Asymptotic and essentially singular solutions of the Feigenbaum equation, J. Stat. Phys. 51 (1988), 991-1007.

[26] J. P. van der Weele, H. W. Capel, and R. Kluiving, Period doubling in maps with a maximum of order $z$, Physica A 145 (1987), 425-460.

[27] M. A. Zaks, Scaling properties and renormalization invariants for the "homoclinic quasiperiodicity", Physica D 62 (1993), 300-316. 